Polymer Journal, Vol. 5, No. 2, pp 107-109 (1973)

\title{
Vibrational Relaxations of Glassy Polymers
}

\author{
Kazuharu Shimizu, Okimichi Yano, and Yasaku Wada \\ Department of Applied Physics, Faculty of Engineering, \\ University of Tokyo, Bunkyo-ku, \\ Tokyo, Japan \\ Yasuaki KAWAMURA \\ Institute of Physical and Chemical Research, \\ Wako, Saitama, Japan
}

(Received February 27, 1973)

\begin{abstract}
KEY WORDS Vibrational / Relaxation / Glassy Polymer / $\gamma$ Peak / Poly(methyl methacrylate) / Polystyrene / Grüneisen Constant /
\end{abstract}

Recently a thermodynamic theory has been developed by Hayakawa, Tanabe, and $\mathrm{Wada}^{1}$ on the dynamic mechanical relaxation in polymers arising from the heat-transfer mechanism between a vibrational degree of freedom and other ones working as a heat bath. The theory gives the relaxation strength $\Delta L$, defined by the increment of instantaneous elastic modulus over the equilibrium one, as

$$
\Delta L=N T \gamma^{2} \varepsilon
$$

Here $N$ is the number of vibrators per unit volume, $T$ is the absolute temperature, $\gamma$ is the Grüneisen constant defined by the derivative of characteristic angular frequency $\omega$ of the vibrator with respect to strain $z$,

$$
\gamma=-\frac{\partial \ln \omega}{\partial z}
$$

and $\varepsilon$ is the specific heat of the vibrator,

$$
\varepsilon=k \frac{x^{2} \exp (x)}{[\exp (x)-1]^{2}}, \quad x=\hbar \omega / k T
$$

where $k$ is the Boltzmann constant.

This report describes the comparison of observed and calculated relaxation strengths for three cases. Since both $\Delta L$ and $\gamma$ in eq 1 depend on the type of deformation, we employ both of them as obtained from longitudinal ultrasonic measurements.

(A) $\alpha$-Methyl Rotation in Poly(alkyl methacrylates)

The mechanical loss peaks of poly(methyl methacrylate), PMMA, which were observed at $115.5^{\circ} \mathrm{K}(46 \mathrm{kHz})$ by Bordoni, Nuovo, and Verdini, ${ }^{2}$ and at $220^{\circ} \mathrm{K}(10 \mathrm{MHz})$ by Tanabe, Hirose, Okano, and Wada, ${ }^{3}$ have been ascribed to $\alpha$-Me rotation ( $\gamma$ peak) because these peaks correlate well with the $T_{1}$ minimum arising from $\alpha$-Me protons in the transition map $^{3}$. A similar loss peak has been found in poly(ethyl methacrylate) and poly(isobutyl methacrylate). ${ }^{3}$ Recent study with neutron inelastic scattering ${ }^{4}$ showed that the rotation of the $\alpha$-Me group of PMMA is excited as an oscillatory motion without being damped by the intermolecular interaction.

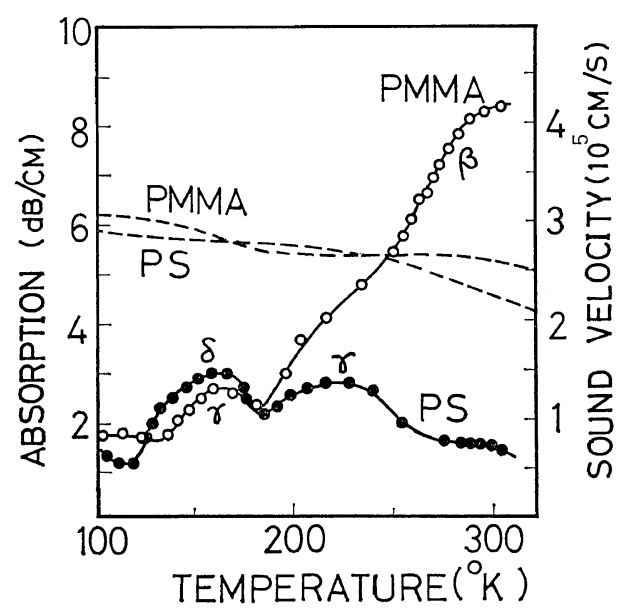

Figure 1. Velocity (dashed lines) and absorption coefficient (open and closed circles) of longitudinal ultrasonic waves at $3 \mathrm{MHz}$ for poly(methyl methacrylate) (PMMA) and polystyrene (PS). 
Figure 1 shows the velocity $u$ and absorption coefficient $\alpha$ of longitudinal ultrasonic waves at $3 \mathrm{MHz}$ in PMMA and polystyrene. The absorption peak of PMMA at $155^{\circ} \mathrm{K}$ is attributed to the $\gamma$ relaxation.

The loss modulus $L^{\prime \prime}$ is evaluated from the equation,

$$
L^{\prime \prime}=\frac{\rho u^{3} \alpha}{\pi f}
$$

where $\rho$ is the density and $f$ is the frequency of the ultrasonic waves. The relaxation strength $\Delta L$ at the loss maximum temperature can be approximately obtained by the equation, ${ }^{5}$

$$
\Delta L=\frac{2 \Delta H}{\pi R} \int L^{\prime \prime} \mathrm{d}(1 / T)
$$

where $\Delta H$ is the activation energy and $L^{\prime \prime}$ is the loss modulus after subtraction of the background loss. The value of $\Delta L$ of the $\gamma$ relaxation of PMMA in Figure 1 is evaluated from eq 5 as $\Delta L=7.9 \times 10^{8} \mathrm{dyn} / \mathrm{cm}^{2}$ (at $155^{\circ} \mathrm{K}$ ), using the activation energy $\Delta H=3.0 \mathrm{kcal} / \mathrm{mol}$. The angular frequency of the $\alpha$-Me rotation is calculated from the moment of inertia of the $\mathrm{Me}$ group and the three-fold potential, $V=\left(V_{0} / 2\right)$ $(1-\cos 3 \varphi)$. In the case of the $\alpha$-Me group, the barrier height $V_{0}$ is reported as $6.4 \mathrm{kcal} / \mathrm{mol}^{3}$, yielding $\omega=6.2 \times 10^{13} \mathrm{rad} / \mathrm{sec}$. Since $\alpha$-Me groups are extruded outside the main chain helix and two Me groups, each belonging to neighbor chains, form a coupled oscillator, $N$ in eq 1 should be taken as the number of such pairs. ${ }^{1}$ The interchain potential is primarily determined by the interaction between such a pair of $\alpha$-Me groups and consequently the Grüneisen constant of the $\alpha$-Me rotation must approximately equal that of the bulk polymer, which was obtained as 4 from the pressure dependence of the longitudinal ultrasonic velocity. ${ }^{6}$ Substituting these values into eq 1 , we obtain the theoretical value of $\Delta L=6.2 \times 10^{8} \mathrm{dyn} / \mathrm{cm}^{2}\left(155^{\circ} \mathrm{K}\right)$, which agrees reasonably well with the observed value.

\section{(B) Ester-Methyl Rotation in Poly(methyl meth- acrylate)}

The $T_{1}$ minimum arising from rotation of ester-Me protons in PMMA was observed at $75^{\circ} \mathrm{K}(21.6 \mathrm{MHz})$ by Powles, Hunt, and Sandiford. ${ }^{7}$ Dynamic mechanical experiments at low frequencies, on the other hand, have not suc- ceeded in detecting a distinct loss peak. ${ }^{8}$ Crissman, Sauer, and Woodward ${ }^{9}$ reported an upswing of loss below $20^{\circ} \mathrm{K}$ and a weak loss peak at $6^{\circ} \mathrm{K}(9.4 \mathrm{kHz})$. The complex shear modulus of PMMA at $34 \mathrm{kHz}$ was measured down to $4^{\circ} \mathrm{K}$ with the composite oscillator method $^{10}$ and is illustrated in Figure 2, where

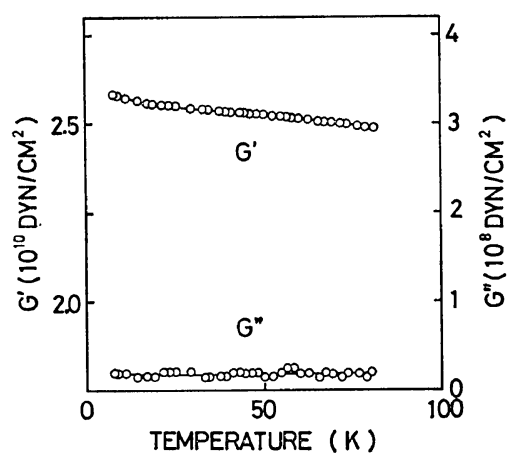

Figure 2. Real and imaginary parts of the complex shear modulus at $34 \mathrm{kHz}$ for poly(methyl methacrylate).

no loss peak is observed down to $4^{\circ} \mathrm{K}$. We measured the ultrasonic absorption at $3 \mathrm{MHz}$ down to $4^{\circ} \mathrm{K}$ but no discernible peak was found.

Since the barrier height $V_{0}$ is $2.4 \mathrm{kcal} / \mathrm{mol}$ for the ester-Me group, ${ }^{3}$ we have $\omega=3.7 \times 10^{13} \mathrm{rad} /$ sec. Assuming $\gamma=4$, we obtain from eq $1 \Delta L=$ $6.4 \times 10^{4} \mathrm{dyn} / \mathrm{cm}^{2}$ at $20^{\circ} \mathrm{K}$, for example, which is so small that the peak, if any, would merge into the backround loss. The apparent weak loss peak found by Crissman, et al., ${ }^{9}$ at $6^{\circ} \mathrm{K}$ may arise from another origin. In fact, if this loss peak temperature is correlated to the $T_{1}$ minimum in the activation plot, we obtain an anomalously low value of activation energy. Some authors ${ }^{11}$ attribute this to the tunneling effect at low temperatures, but according to Tanabe, et al., ${ }^{3}$ the tunneling effect is not effective in the mechanical relaxation.

\section{(C) Phenyl Rotation of Polystyrene}

The $\gamma$ peak of polystyrene which was observed at $132^{\circ} \mathrm{K}(1 \mathrm{~Hz})$ by Illers and Jenckel ${ }^{12}$ and at $200^{\circ} \mathrm{K}(34 \mathrm{kHz})$ by Yano and Wada ${ }^{10}$ was ascribed by Yano and Wada to the rotation of the phenyl group about the $\mathrm{C}-\phi$ bond. This view is supported by the fact that this relaxation is inactive in dielectric measurements, even for 
poly-parafluorostyrene. The activation energy of the relaxation is $9.0 \mathrm{kcal} / \mathrm{mol}^{10}$ and the relaxation strength is $\Delta L=1.1 \times 10^{9} \mathrm{dyn} / \mathrm{cm}^{2}$ at $220^{\circ} \mathrm{K}$, from the data in Figure 1. The potential of rotational vibration is calculated, assuming a $3_{1}$ helix conformation of the main chain, from the van der Waals interaction between two $\mathrm{CH}$ groups at the ortho position in the phenyl ring and five carbons and eight hydrogens in the main chain, the latter being located within $5 \AA$ of the former. In this calculation, the parameters of the pair-wise potential between $\mathrm{C}-\mathrm{C}$, $\mathrm{C}-\mathrm{H}$, and $\mathrm{H}-\mathrm{H}$ were taken from Scott and Scheraga. ${ }^{13}$ The characteristic frequency of the rotational vibration of the phenyl group was obtained from this potential as $\omega=9.7 \times 10^{12} \mathrm{rad} /$ sec, which is similar to $\omega=13 \times 10^{12} \mathrm{rad} / \mathrm{sec}$ obtained by Reich and Eisenberg ${ }^{14}$ for a much simpler model. This value, along with $\gamma=4^{6}$ and $N$ as the number of phenyl pairs, yields $\Delta L=1.5 \times 10^{9} \mathrm{dyn} / \mathrm{cm}^{2}$ at $220^{\circ} \mathrm{K}$ from eq 1 which compares well with the experimental value.

The relaxation time of the vibrational relaxation is determined by the rate of heat-transfer between the vibrational mode and the other modes working as a heat bath. The latter are for the most part vibrational modes of the main chain and the heat-transfer arises from anharmonic coupling among vibrations. Therefore, mechanical loss maxima due to vibrational relaxations do not necessarily coincide exactly with $T_{1}$ minima in the activation plot, and the activation energy of the mechanical relaxation is in general different from the potential barrier for the rotation.

\section{REFERENCES}

1. R. Hayakawa, Y. Tanaba, and Y. Wada, $J$. Macromol. Sci., (in printing).

2. P. G. Bordoni, M. Nuovo, and L. Verdini, Nuovo Cimento, 20, 669 (1961).

3. Y. Tanabe, J. Hirose, K. Okano, and Y. Wada, Polymer J., 1, 107 (1970).

4. J. S. Higgins, G. Allen, and P. N. Brier, Polymer, 13, 157 (1972).

5. N. G. McCrum, R. E. Read, and G. Williams, Anelastic and Dielectric Effects in Polymeric Solids, John Wiley, London, 1967.

6. Y. Wada, A. Itani, T. Nishi, and S. Nagai, J. Polym. Sci., Part A-2, 7, 201 (1969).

7. J. G. Powles, B. I. Hunt, and D. J. H. Sandiford, Polymer, 5, 505 (1964).

8. K. M. Sinnott, SPE Trans., 2, 65 (1962).

9. J. M. Crissman, J. A. Sauer, and A. E. Woodward, J. Polym. Sci., Part A-2, 5075 (1964).

10. O. Yano and Y. Wada, J. Polym. Sci., Part A-2, 9, 669 (1971).

11. J. A. Sauer, ibid., Part C, 32, 69 (1971).

12. K. H. Illers and E. Jenckel, ibid., 41, 528 (1959).

13. R. A. Scott and H. A. Scheraga, J. Chem. Phys., 42, 2209 (1956); 44, 3054 (1966); 45, 2091 (1966).

14. S. Reich and A. Eisenberg, J. Polym. Sci., Part A-2, 10, 1397 (1972). 